\title{
The disciplines of management and IT have indeed merged: new empirical data
}

Advancement in the intelligence field can only be achieved through new observations and the presentation of new empirical data. This is a continuous process and includes how we as employees engage with software and technical solutions. Just as it is impossible to teach or learn anything in marketing today without a deep understanding of digital marketing, in the same way is it impossible to make advancements in intelligence studies without first-hand experience with business intelligence software and new IT-equipment. Management and IT have indeed merged.

This understanding has been an integrated part of JISIB since the journal started some eight years ago. And as always, we are less interested in how new technologies are developed (for that there are excellent technical journals) than about the management practice of these developments. This issue follows very much on this track.

The article by Fatma Fourati-Jamoussi, Claude-Narcisse Niamba and Julien Duquennoy entitled "An evaluation of competitive and technological intelligence tools: A cluster analysis of users' perceptions" is an evaluation of competitive and technological intelligence (CTI) tools by students to help designers get the best efficiency out of a monitoring process. The paper finds that user perception is greater than expected and that designers of CTI tools must take this in account when developing new products. The authors argue that this is a major reason why new software implementation fails in organizations.

The article by Ahmad Abbaspour, Amir Hussein Amirkhani, Ali Asghar Pour Ezzat, and Mohammad Javad Hozori is entitled "Identifying and describing sub-processes in strategic intelligence process by qualitative content analysis in inductive way". The authors set out to identify and describe the sub-processes of the strategic intelligence process in organizational analysis. Fourteen main sub-processes are identified to describe the strategic intelligence process. The results give new insight into the strategic intelligence process implementations in organizations.

The article by Mourad Oubrich, Abdelati Hakmaoui, Robert Bierwolf and Mouna Haddani entitled "Development of a competitive intelligence maturity model-Insights from Moroccan companies" identifies six CI dimensions (CI culture of an organization, CI deliverables, CI sourcing, CI cycle, CI investment in terms of resources, CI users and CI application) in CI implantation at three different CI levels (early, mid, world class).

The article by Avner Barnea entitled "Israeli start-ups - especially in cyber: can a new model enhance their survival rate?" concludes that the high percentage of failures of Israeli start-ups is due to the difficulties in comprehending the competitive landscape. Barnea draws this conclusion from having worked and interviewed a number of companies for years. He introduces what he calls the competitive review model to help small companies better prepare themselves for intense competition, especially relevant for the cyber security industry.

This issue also features a book review of Tetlock and Gardner's Superforecasting: The art and science of prediction (2015, Crown Publishers, New York, NY).

As always, we would above all like to thank the authors for their contributions to this issue of JISIB. Thanks to Dr. Allison Perrigo for reviewing English grammar and helping with layout design for all articles and to the Swedish Research Council for continuous financial support.

On behalf of the Editorial Board,

Sincerely Yours,

$$
\text { Krim 1. Nhin }
$$

Prof. Dr. Klaus Solberg Søilen Halmstad University, Sweden Editor-in-chief 